ISSN 1112-9867

\title{
HOW CAN PATIENT JOURNEY IN SURGICAL WARDS OF A REFERRAL HOSPITAL BE IMPROVED?
}

\author{
F. Rezaei ${ }^{1}$, M. H. Yarmohammadian ${ }^{2}$, Y. Molavi Taleghani ${ }^{1}$, F. Ghorat ${ }^{3}$, B. Alizadeh $^{4}$, M. \\ Vejdani $^{5^{*}}$
}

\begin{abstract}
${ }^{1}$ Phd Student, Department of health management and medical informatics, Health Management and Economics Research Center, Isfahan University of Medical Sciences, Isfahan, Iran

${ }^{2}$ Professor, Health in disasters and Emergencies, Health Management and Economics research center, Isfahan University of Medical Science, Isfahan, Iran

${ }^{3}$ Traditional and Complementary Medicine Research Center, Sabzevar University of Medical Sciences, Sabzevar, Iran

${ }^{4}$ Resident of Emergency medicine, Department of Emergency Medicine, Faculty of Medicine, Mashhad University of Medical Sciences, Mashhad, Iran

${ }^{5}$ Iranian Research Center on Healthy Aging, Sabzevar University of Medical Sciences, Sabzevar Iran
\end{abstract}

Published online: 15 February 2017

\begin{abstract}
Background: We studied the patient journey in surgical wards in order to find an effective and efficient way of scheduling in surgical wards.

Methods: We applied Root cause analysis (RCA) model within three months in a referral hospital. After understanding root causes of the events occurred through a focus discussion group, required interventions were proposed according to literatures, experiences, and preference of the stakeholders. Possible interventions were also analyzed based on its ability to reduce contributing factors in the events and the belief of process-owner that if interventions can be implemented.
\end{abstract}

Author Correspondence, e-mail: author@gmail.com

doi: http://dx.doi.org/10.4314/jfas.v9i1s.717 
Results: The results were provided for five main steps: 1) the most important root cause was "not prioritizing patients and pre-scheduling the number of surgical procedures in the days before". 2) Constraints indicated that workforce weren't allocated proportionally to the number of surgical operations in varying shift lengths, increased numbers of on-calls physicians increased related costs, the admission of patients in VIP wards have been getting a high priority, and surgeon compensation based on fee for service method was challenging. 3) The current situation of allocating three rooms on average for each physician can be changed depending on numbers of surgeries. 4) Proposed interventions are establishing a computer registration system, reforming payment methods, setting up an electronic waiting list, development of scheduling guidelines, and Applying MIP model.

Conclusions: Implementing of scheduling reforms requires a comprehensive action plan system and predefined functional indicators. These should be achieved with considering comments of all clinical and technical groups to ensure the feasibility of an operating room schedule.

Keywords: patient journey, surgery, scheduling, Root cause analysis (RCA), patient transfer Key messages:

- $\quad$ Scheduling in the surgical wards has a critical effect on surgical procedures. An electronic based registry software system is required at least in our country. This system should prepare in the form of dashboard management tool with predefined criteria.

- Development of such this system needs cooperation of both clinical and IT staff. Physicians are the most important key stakeholders to participate.

Acknowledgments: The authors are grateful to the Chief Executive and to Surgery Department personnel of the Teaching Hospital, Iran, for their active collaboration and for sponsoring the present research, and to Ms. Azary and Ms. Hsseiny for contributing to the development of this Study.

BACKGROUND: hospitals are always looking for cost reduction solutions, improving financial assets, andincreasingpatient's satisfaction and quality (1). A ward in a hospital, which needs to be paid attention specifically is the surgical ward since these facilities are the largest cost and income centers of a hospital and impact on the performance of the entire hospital(2). In addition, management of surgery department is so difficult not only for various preferences of 
stakeholders, but also for insufficient resources in developing countries $(3,4)$. In addition, health managers should anticipate the impact of the aging population on increased demand for surgical services $(5,6)$. The above factors clearly show the importance of having an efficient scheduling procedure for surgery in order to allocate the proper time to proper service(7).

A large part of the past studies have investigated this issue and proposed several interventions. In general, researchers provided two styles of scheduling included:1) advance scheduling and 2) allocating scheduling procedures. Advance schedules specify a certain surgery date for each patient while allocating schedules determine a specific room, day and time for surgery procedure. But, recently efforts are focused more on the integration of two approaches $(8,9)$. In addition, other researchers have added a new area to scheduling by which identifying and reserving all external resources and coordination with other department are required $(10,11)$. In Brecht Cardoen et al study, three major goalswere: First, updating scheduling of surgery wards base on the last researches and developed methods in this area of science. Secondly, information management in order to make all practitioners have effective communications and investigate various aspects of scheduling. Third, integrating achieved sciences in this area accurately to identify issues that have not been investigated so far(2).

AIMS: In the study, we have surveyed possible strategies for scheduling comprehensively. Then requirements of a standard schedule determined based on critical criteria and functional indicators. In our hospital, decision makers of the most responsible departments in surgical procedures selected the most applicable intervention. Finally, the target intervention was determined in a certain five-step process.

\section{METHODS}

We used the RCA model to narrate chronology events during three months in a referral hospital in Iran. Then, root causes of the events were determined by process owners in FDG meetings. Eight meetings were held. Each lasted two-hour. Process owners/ stakeholders werethe educational supervisor, head nurse of operating room, head nurse of anesthesia team, hospital risk manager, recovery head nurse, day- clinic head nurse and head nurse of other departments if necessary and those were responsible for hospital accreditation.

Contributing factors (root causes) in each event were prioritized based ontheir role and importance in the occurrence of the current event (ranked 1-5) and future events (ranked 1-5) 
(12). In the next stage, constraints and problems arising from those root causes were discussed in meetings. We also interviewed separately with key process owners. Content analysis was done to extract key concepts. Proposed interventions were accounted bothexperiences of process owners andliteratures. We aimed to developapplicable reforms.

These interventions should be analyzed in the hospital based on 1) ability to mitigate the contributing factor 2) team's belief that the intervention will be implemented and executed (12).

\section{RESULTS}

We divided results into five main steps. These sections included root causes identification, the constraints, and problems due to root causes, the current procedure in allocating days and rooms tosurgeons, FDG member recommended interventions and intervention in priority for the hospital in our study.

1. We considered causal roots of reported events during 3 months based on content analysis and agreement between all members of the FDG. The most important causal roots were:not having waiting list and schedulingfor number of surgeries in advance (even the day before), surgeons don't informhead nurses (wards) of priority of patients that should be operated, the large number of surgeries (more than eighty in one day), and pre-determinedsurgery numbersare considered an important issue.On the other hand, number of surgeries would beannouncedevery morning to surgery ward head nurse.

2.Constraints and problems arising from root causes which were inducted by interviews:

2.1. Disproportion of the workforce, equipment and facilities to the number of surgeries

2.2. Disproportion of working hours dedicated to surgeonstothe number of their surgeries in a specific day.

2.3. Physician's order for a surgery would not be provided to the hospital in advanced. Patientsare referred to the hospital after getting the physician's orders forhospital admission (without precoordination with the hospital)

2.4. Some patientshave two orders from two surgeons for admission simultaneously

2.5. Increased number of on-call physicians would cause induced cost of the hospital due to the increased number of patients in a day. 
2.6. Head nurse of the surgery ward would not be informed aboutcauses of physician delay or absence in the allocated days. So,surgical bed and workforce don't have enough efficiency at those hours.

2.7. Possible delays in the operating rooms don't considerwhen admitting patients.

2.8. There are no plans and schedules for patient referredfrom private sector patients against public sector patients

2.9. Giving priority in admission of patients referred from private sector and delaying patents referred from public sector (for more revenue)

2.10. Unawareness of other departments of hospital of number of surgeries of each physician in same day, the number of waiting people in the admitting of surgical ward, number of reserves, number of emergency surgeries and number of canceled surgeries.

2.11. Surgeons compensation based on the fee for service method

2.12. The direct relationship between the hours allocated to each surgeon in an operating room with the number of their surgeries (figure1)

2.13. Large number of surgeries without waiting list led to operating out of turn. (Surgeons out to presentin days allocated to other physicians)

3. Current situation in allocation of days and rooms to physicians:

In the studied hospital, allocation of hours/days of operating rooms to surgeons is based on the numbers of their surgeries in three/six months before. This means that physicians who operatemore surgeries would be given more resource.

Figure 1- Comparative analysis of hours allocated to pediatric professors in the operating rooms in first six months of 2015

As seen, in the above chart, physician $\mathrm{C}$ had less number of surgeries. As a result, in most cases, operation rooms allocated to him/herhad been left empty. While physician A had higher number of surgeries and he/shewould probably be faced with problem to perform all his surgeries. Therefore, days allocated to Physician C should be reduced and added to Physicians A and B. According to FDG member's statements, three rooms have been allocated on average to each physician, while it could be varied depending on the numbers of their surgeries. When a physician is absent in a certain day, according to head nurse discretion, the room will be allocated to other physicians who have more patients in the waiting room and also have more preparedness (in terms of patient preparedness, equipment availability, adequate staffing, etc). Finally, this 
scheduling should be updated by entering new professors. The information should be entered in HIS system.

4- Proposed interventions:

1. Establishing a registry system for surgeries in HIS.The system should have a specific user and password for each surgeon. So, they can register their surgeries in advance.

2. Improving surgeon's payment methods, both based on fee for services and salary.

3. Developing an Electronic Waiting List in whicha surgical case duration can be predicted (based on V.S of patient, time needed for anesthesia before incision, the time needed to place a catheter in the bladder, or the wake-up time from anesthesia, previous experiences for each surgery and standards/ nonstandard surgeries). The FDG memberspreferred to apply designs developed in foreign countries.

4. Developingguidelinesforscheduling according to five following criteria. That's the responsibility of the director of operating rooms and educational deputy to determine key indicators in the form of dashboard management tool. The aim is to indicate status of operating room at the moments. In addition, dashboard management tool should be run in HIS system.

\section{- $\quad$ Patient's Characteristics:}

a) Elective or Non- Elective - In our study, the investigated surgery ward was elective. But,we considered this criterion since emergency patients would be transferred sometimes to elective ward due to completion of emergency OR capacity.

b) Outpatient vs. inpatient surgery

\section{- $\quad$ Functional measurement:}

Waiting time, Preferences of each clinical group in the OR, resource utilization, OR throughput, leveraging of resources, risk reduction inOR capacityexpansion, patient deferral, makespan, time interval between the first patient entrance and last patient exit from surgery ward, the number of surgery cancellations and delays, financial objectives with regard to the impact of operating room scheduling in cost saving

\section{- Decision delineation:}

This indicates decision making about the date, time, and operating room allocations to surgical specialties and clinical groups. For example, in our study, days and roomsareallocated to physicians depending on requirements for their surgery. 


\section{- Constraints:}

The capacity of holding area/ Post- anesthesia care unit, inpatient wards, ICU, recovery room, equipmentconstraints, shortages of personnel, budget,limitation ofavailable time, OR overtimes constraints on predicting demands andexact date for surgeries and sharing personnel preferences in OR.

\section{- Uncertainty:}

This criterion applies in some situation. For example, delays in starting surgical procedures, the emergency patient admission due to lack of capacity in emergency operating rooms, increased length of surgery compared to the predicted time.

5- Use of Mixed Integer Programming (MIP) model developed by Iranian researchers considering the limitations of the third level educational hospitals in Iran (applicability of this research for our educational referral hospital) (13).

5. Prioritizing interventions based on two criteriaof 1) ability to mitigate the contributing factor, and 2) team's belief that the intervention will be implemented and executed(14).

Table 1: Prioritizing interventions based on FDG member suggestion

Figure 2- Overview the study process and results

Most of limitations related to planning and scheduling of working hours in the operating rooms can be resolved by five criteria of intervention number Four. But, FDG members suggested integration of these criteria into a registry system for surgeries in HIS. In this system, constraints and thresholds for each criterion are predefined automatically. To achieve this goal, requirements included: 1) the commitment of surgeons to the rules and 2) admission of surgeries through such a registry system.

\section{DISCUSSION}

In our hospital, current decisions are made at the physician level. This means thatfor decision delineation criterion, allocated capacity (date, time, and operating room) should be definedat the physician level. There are three decision-making levels included patient, physician, and surgical disciplines or specialties. At the surgical specialty level, researchers provided a model in which specified days and ORs were allocated to each discipline or clinical groups by developing timetable cycle. We can do as Beliën et al to integratetwo selected interventions by designinga software in which specified days and ORs were allocated to each surgeon/physician(15). In this 
case, each surgeon performs based on a predefined protocol to reserve a certain time, date, specified room and other items. If average time of each surgery and the capacity of ORs in a certain day had beenpredefined in the registry system, physicianscouldregister surgeries only in accordance with available capacities.In addition, if length of stay in ORs increased, fewer surgeries would be performed by surgeons(16). In the Van Berkel and Blake study, input analysis was done to reduce wait time (17). So, resource utilization would actually happen when cancellation of planned surgeries and overtime cost reduced. On the other hand, we will not be faced with over- admitted patients in surgery ward. These issues are discussed in operation research studies(18). You can imagine overcrowding in an operating room, while the others are not utilized because they were allocated to certain specialties. This issue is known as affinities among surgical teams(19). Finally, at the third level of decision making "based on patient criteria", type of admission (elective or non-elective), outpatient vs. inpatient surgery, and surgical procedures are considered to specify times and operating rooms.

For constraints criterion, capacities of hospitalization wards, recovery, ICU and holding area are substantial.As patient journey is a continuous process from admission in the surgical ward to transference to a hospitalized ward(20),any interruption of the process, will increase waiting time and cause inefficiencies of surgeon's performance. Events caused by lack of coordination, lack of communication and information sharing(21). In this case, a registrysystem should be designed in a way that if ICU capacity strained of itscritical care capacity, admission of additional patients would be prevented automatically. Additionally, reserving OR capacity for emergency patients should always be considered. Wullink et al have recommended that critical care capacity should be allocated to all types of operating rooms not only one operating room for emergency patients. This increases accountability, utilization and more accessibility and timesaving (22). So, we canuse literatures toachieve operational knowledge and optimize software. In addition, requirements should be determined for public and private patients' priorities in allocation of the ORs. So, right of public patients would be kept.

It should be noted that there are some policies, constraints, capacities in each hospital. It's necessary to consider all these when a software is going to be developed with some registry criteria. According to Patterson, three types of general strategies are used for scheduling in the surgical department:

1- Blocked planning strategy 
In this strategy, a timetable (usually weekly or monthly) is given to surgeons (surgical teams). Patient-surgery time is allocated in timetable (Time Block). But, this strategy makes two questions: first, how should be a periodic timetable developed? And, how should be specialized ORs and times allocated to each surgical discipline. Such timetable is called Master Surgical Schedule (MSS). The second one is how OR times allocated to surgeons match with each required time of all registered cases. If these times do not match automatically, surgeons might demand extra capacity.

2. Open planning strategy

In this strategy, surgery cases are allocated to available operating rooms based on surgeons' preferences. Free times in the plan will be filled according to First Come First Served (FCFS) approach. In this approach, services are provided for those who have registered earlier in chronological order. Scheduling is based on allocation of rooms to each surgery one day before the surgery.

\section{Reformed planning strategy}

In this approach, blocked scheduling strategy can be reformed in two ways to improve flexibility. First, reserving any number of time blocks and then, announcing the free times of each OR exactly before surgery (18).

We used reformed planning strategy. We allocated an operating room to each surgeon in any day of the week. This follows blocked planning strategy. However, we used open planning strategy in allocation of times to different surgical cases of each surgeon. In this approach, the numbers of surgical cases were determined in everyday morning and head nurse reorganized the operating rooms in coordination with other surgeons when any of them becamefree.However, we confronted with lots of conflicts between clinical disciplines and that's the reason for doing this research. We mainly focused on blocked planning strategy.Surgeonshave the responsibility to register patients in the system. There should be some predefined limitations in the software (for example, the number of surgeries limited by the average time spent to each surgery). Finally, inpatient wards can be informed ofthe schedules. Some degree of flexibility should be considered for surgeon and they should not be forgotten as key stakeholders in providing such software.

The second intervention titled "reforming pay practices based on salaries rather and fee for service «was confirmed as other hospitals applied and had good results. This intervention is a motivational-financial solution.In our hospital monthly statistics of frequency of the surgeries are 
determinants of time allocation to surgeon. But, that's a retrospective approach to solving the problem.

In third intervention, the possible duration of each surgery would be determined based on type of surgeries (which represents the average time needed to allocate operating rooms) and V.S. of patients in the software(23). Although the intervention is effective and useful to improve scheduling, but being informed of the number of surgeries in each day is aprerequisite to provide a waiting list. Therefore, both average times needed for each surgery and approximate number of surgeries should be considered to developa comprehensive plan.

\section{CONCLUSION}

Each intervention has some advantages and disadvantages. The important issue should beapplyingan effective and practical solution to improve throughput, utilization, income, clinical effects, staff overtime, recovery care and patient satisfaction (24). However, proper use of these criteria in the selected strategy depends oninvolvingtwo groups of experts in designing software: clinical and non-clinical (25).

An important problem in root cause analysis wasthe team composed mostly of health service providers. The team could nothavean expert view on non-clinical issues such as human factor engineering, organizational sociology and psychology (organizational behavior), and medical instrument manufacturers. On the other hand, the team faced with political and economic problems during the implementation the selected strategy. Team members were unaware of the resources needed to implement the strategies. Some interventions require additional human resource as the most expensive resource. In addition,coordination with other departments is necessary when problems have originated fromanother department. The quality of the software for the selected strategy should not be reduced due to time constraints of accreditation(14).Therefore, when scheduling softwareis going to be developed, it is necessary to involve important interest groups and stakeholdersto investigate costs, feasibility, responsibilities, required time etc.

Finally, acceptability of these methods is always under question by clinical staff. Therefore, researchers have questioned recommendations without executive guarantee in the risk evaluation models. Assurance the applicability of interventions needsa comprehensive action plan and performance measurements. Technical comments of all stakeholders should also be used 
throughout planning. Fortunately, technological developments have made it possible to implement low-cost and effective solutions.

\section{REFERENCES}

1. Parand A, Dopson S, Renz A, Vincent C. The role of hospital managers in quality and patient safety: a systematic review. BMJ open. 2014;4(9):e005055.

2. Cardoen B, Demeulemeester E, Beliën J. Operating room planning and scheduling: A literature review. European Journal of Operational Research. 2010;201(3):921-32.

3. Glouberman S, Mintzberg H. Managing the care of health and the cure of disease-Part I: Differentiation. Health care management review. 2001;26(1):56-69.

4. Linden AF, Sekidde FS, Galukande M, Knowlton LM, Chackungal S, McQueen KAK. Challenges of Surgery in Developing Countries: A Survey of Surgical and Anesthesia Capacity in Uganda's Public Hospitals. World Journal of Surgery. 2012;36(5):1056-65.

5. Etzioni DA, Liu JH, Maggard MA, Ko CY. The aging population and its impact on the surgery workforce. Annals of surgery. 2003;238(2):170-7.

6. Fraher EP, Knapton A, Sheldon GF, Meyer A, Ricketts TC. Projecting Surgeon Supply Using a Dynamic Model. Annals of surgery. 2013;257(5):867-72.

7. Macario A. Implementing operating room management science: from the bench to the scheduling office. European Journal of Anaesthesiology (EJA). 2014;31(7):355-60.

8. Magerlein JM, Martin JB. Surgical demand scheduling: a review. Health services research. 1978;13(4):418.

9. Banditori C, Cappanera P, Visintin F. A combined optimization-simulation approach to the master surgical scheduling problem. IMA Journal of Management Mathematics. 2013:dps033.

10. Przasnyski ZH. Operating room scheduling: A literature review. AORN journal. 1986;44(1):67-82.

11. Manolitzas P. Effective Methods for Modern Healthcare Service Quality and Evaluation: IGI Global; 2016.

12. Rockville M. Learning from Defects. John Hopkins: Agency for Healthcare Research and Quality.; 2012. 
13. Ghazalbash S, Sepehri MM, Shadpour P, Atighehchian A. Operating room scheduling in teaching hospitals. Advances in Operations Research. 2012;2012.

14. Pham JC, Kim GR, Natterman JP, Cover RM, Goeschel CA, Wu AW, et al. ReCASTing the RCA: an improved model for performing root cause analyses. American Journal of Medical Quality. 2010;25(3):186-91.

15. Denton BT. Handbook of Healthcare Operations Management: Methods and Applications: Springer New York; 2013.

16. Denton B, Gupta D. A sequential bounding approach for optimal appointment scheduling. Iie Transactions. 2003;35(11):1003-16.

17. VanBerkel PT, Blake JT. A comprehensive simulation for wait time reduction and capacity planning applied in general surgery. Health care management Science. 2007;10(4):37385.

18. Guerriero F, Guido R. Operational research in the management of the operating theatre: a survey. Health care management science. 2011;14(1):89-114.

19. Meskens N, Duvivier D, Hanset A. Multi-objective operating room scheduling considering desiderata of the surgical team. Decision Support Systems. 2013;55(2):650-9.

20. Rezaei F, Yarmohammadian MH, Ferdosi M, Haghshenas A. Principles of risk management in surgery departments. Archives of Clinical and Experimental Surgery (ACES). 2015;4(3):126-34.

21. Al Hakim L, Gong XY. On the day of surgery: how long does preventable disruption prolong the patient journey? International Journal of Health Care Quality Assurance. 2012;25(4):322-42.

22. van Oostrum JM, Van Houdenhoven M, Hurink JL, Hans EW, Wullink G, Kazemier G. A master surgical scheduling approach for cyclic scheduling in operating room departments. OR spectrum. 2008;30(2):355-74.

23. Stepaniak PS, Heij C, Mannaerts GH, de Quelerij M, de Vries G. Modeling procedure and surgical times for current procedural terminology-anesthesia-surgeon combinations and evaluation in terms of case-duration prediction and operating room efficiency: a multicenter study. Anesthesia \& Analgesia. 2009;109(4):1232-45. 
24. Kayis E, Wang H, Patel M, Gonzalez T, Jain S, Ramamurthi R, et al., editors. Improving prediction of surgery duration using operational and temporal factors. AMIA Annual Symposium Proceedings; 2012: American Medical Informatics Association.

25. MacCormick AD, Parry BR. Judgment analysis of surgeons' prioritization of patients for elective general surgery. Medical decision making. 2006;26(3):255-64.

Table 1. Scores of FGD member based on priorities

\begin{tabular}{|c|c|}
\hline $\begin{array}{c}\text { Recommended } \\
\text { interventions }\end{array}$ & Scores \\
\hline Intervention 1 & 72.14 \\
\hline Intervention 2 & 5.19 \\
\hline Intervention 3 & 48.41 \\
\hline Intervention 4 & 81.33 \\
\hline Intervention 5 & 8.9 \\
\hline
\end{tabular}

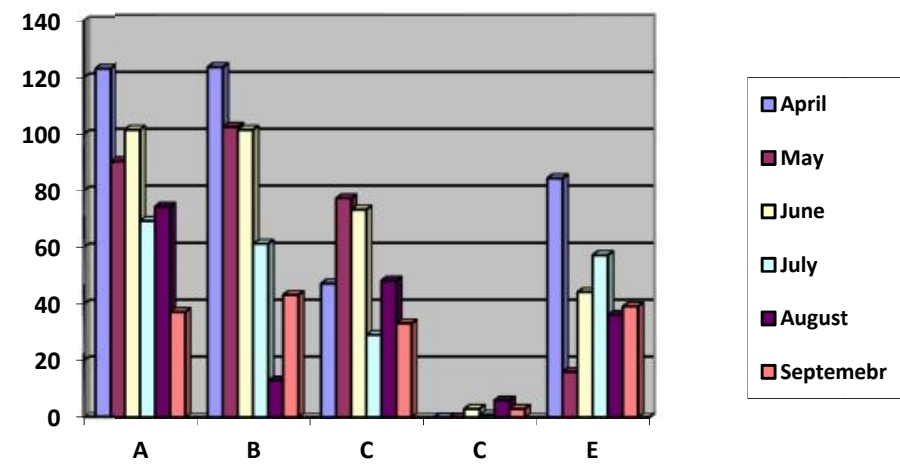

Fig.1. Comparative analysis of hours allocated to pediatric professors in the operating rooms in first six months of 2015 


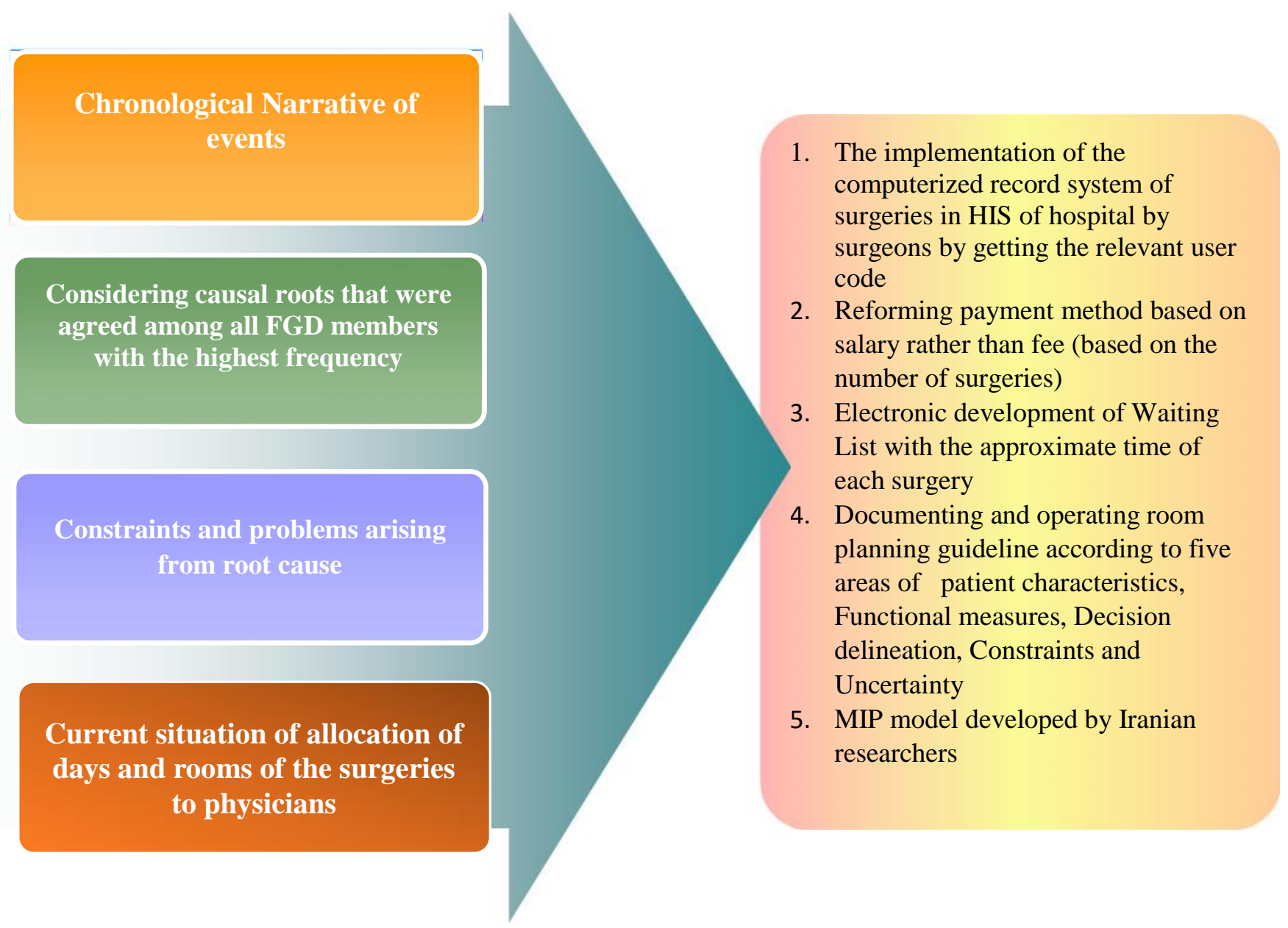

Fig.2. Overview the study process and results 
How to cite this article:

Rezaei F, Yarmohammadian M H, Molavi Taleghani Y, Ghorat F, Alizadeh B, Vejdani M. How can patient journey in surgical wards of a referral hospital be improved?. J. Fundam. Appl. Sci., 2017, 9(1S), 647-661. 